\title{
Sodium-Glucose Cotransporter 2 Inhibitors: Possible Anti- Atherosclerotic Effects Beyond Glucose Lowering
}

\author{
Hidekatsu Yanaia, b, Hisayuki Katsuyama $^{\text {a }}$, Hidetaka Hamasaki ${ }^{a}$, Hiroki Adachi ${ }^{\text {a }}$ \\ Sumie Moriyama ${ }^{\text {a }}$, Reo Yoshikawa ${ }^{a}$, Akahito Sako ${ }^{a}$
}

\begin{abstract}
The new drug for type 2 diabetes, the sodium-glucose cotransporter 2 (SGLT-2) inhibitor, is reversible inhibitor of SGLT-2, leading to reduction of renal glucose reabsorption and decrease of plasma glucose, in an insulin-independent manner. In addition to glucose control, the management of coronary risk factors is very important for patients with diabetes. Here we reviewed published articles about the possible anti-atherosclerotic effects beyond glucose lowering of the SGLT-2 inhibitors. We searched by using Pubmed, and found 770 published articles about SGLT-2 inhibitors. Among 10 kinds of SGLT-2 inhibitors, the number of published articles about dapagliflozin was the greatest among SGLT-2 inhibitors. Since SGLT-2 inhibitors have similar chemical structures, we concentrated on the published articles about dapagliflozin. SGLT-2 inhibitors are proved to be significantly associated with weight loss and reduction of blood pressure by a relatively large number of studies. The studies investigating effects of dapagliflozin on visceral fat, insulin sensitivity, serum lipids, inflammation and adipocytokines are very limited. An influence of increase in glucagon secretion by SGLT-2 inhibitors on metabolic risk factors remains unknown.
\end{abstract}

Keywords: Atherosclerosis; Blood pressure; Body weight; Glucagon; Sodium-glucose cotransporter 2 inhibitor

\section{Introduction}

Sodium-glucose cotransporter 2 (SGLT-2) mediates approximately $90 \%$ of active renal glucose reabsorption in the proximal tubule of the kidney [1]. Recently, the new drug for type 2 diabetes, the SGLT-2 inhibitor was developed. The SGLT-2 inhibitor is reversible inhibitor of SGLT-2, leading to reduction of renal glucose reabsorption and decrease of plasma glucose,

\footnotetext{
Manuscript accepted for publication October 23, 2015

aDepartment of Internal Medicine, National Center for Global Health and Medicine Kohnodai Hospital, Chiba, Japan

${ }^{b}$ Corresponding Author: Hidekatsu Yanai, Department of Internal Medicine, National Center for Global Health and Medicine Kohnodai Hospital, 1-7-1 Kohnodai, Chiba 272-8516, Japan. Email: dyanai@hospk.ncgm.go.jp
}

doi: http://dx.doi.org/10.14740/jocmr2385w in an insulin-independent manner [2]. Diabetes is a strong independent risk factor for cardiovascular diseases (CVDs) [3]. Compared with subjects without diabetes, the relative risk for CVD is 2 - 3 times greater in men with diabetes and 3 - 4 times greater in women with diabetes [4-10]. In addition to glucose control, the management of coronary risk factors is very important for patients with diabetes. Here we reviewed published articles about the possible anti-atherosclerotic effects beyond glucose lowering of the SGLT-2 inhibitors.

\section{The Search Strategy for Published Articles About the Anti-Atherosclerotic Effects Beyond Glucose Lowering of the SGLT-2 Inhibitors}

We searched by using Pubmed (Table 1), and found 770 published articles about SGLT-2 inhibitors. Ten kinds of SGLT-2 inhibitors were detected, and we searched the published articles about each SGLT-2 inhibitor. The number of published articles about dapagliflozin was the greatest among SGLT-2 inhibitors. Since SGLT-2 inhibitors have similar chemical structures, we concentrated on the published articles about dapagliflozin.

\section{Glucose, Body Weight and Blood Pressure Lowering Effects of Dapagliflozin}

Dapagliflozin also reduces renal glucose reabsorption and decrease of plasma glucose, in an insulin-independent manner [2], which induces reduction of body weight and blood pressure. Reduction of body weight and blood pressure by SGLT-2 inhibitors is also induced by osmotic diuretics [11]. There were 106 published articles about "dapagliflozin and body weight" and 78 articles about "dapagliflozin and blood pressure".

Matthaei et al studied effects of dapagliflozin $10 \mathrm{mg} /$ day or placebo for 52 weeks on metabolic parameters in patients with type 2 diabetes using sulphonylurea and metformin [12], $\mathrm{HbAlc}$ and fasting plasma glucose levels showed greater improvement from baseline with dapagliflozin $(-0.8 \%$ and -1.5 $\mathrm{mmol} / \mathrm{L}$ ) than with placebo. Dapagliflozin was associated with greater reductions in body weight and systolic blood pressure (-2.9 kg and -1.0 mm Hg) compared with placebo. Dapagliflozin was administered as monotherapy $(\mathrm{n}=249)$ or combination 
Table 1. The Reported Sodium Glucose Cotransporter 2 Inhibitors

\begin{tabular}{ll}
\hline The search strategies by Pubmed & Published articles (n) \\
\hline $\begin{array}{l}\text { Sodium glucose cotransporter } \\
2 \text { inhibitor OR sodium glucose } \\
\text { cotransporter } 2 \text { inhibitors OR } \\
\text { SGLT2 inhibitor OR SGLT2 } \\
\text { inhibitors OR SGLT-2 inhibitor }\end{array}$ & 770 \\
OR SGLT-2 inhibitors & \\
Each sodium glucose \\
cotransporter 2 inhibitors
\end{tabular}

therapy $(n=479)$ with existing antihyperglycemic agents to Japanese patients with diabetes for 52 weeks [13]. In patients receiving dapagliflozin as monotherapy or combination therapy, reductions from baseline were observed in $\mathrm{HbA} 1 \mathrm{c}(-0.7 \%$ in both groups), weight (-2.6 and $-2.1 \mathrm{~kg}$, respectively), and systolic blood pressure (-5.2 and $-3.9 \mathrm{~mm} \mathrm{Hg}$ ). Dapagliflozin reduced body weight and blood pressure by both monotherapy and add-on therapy.

In a meta-analysis including all trials with a duration of at least 12 weeks, comparing an SGLT-2 inhibitor with a nonSGLT-2 inhibitor agent in type 2 diabetes, SGLT-2 inhibitors are effective in the treatment of type 2 diabetes, providing additional benefits, such as weight loss, reduction of blood pressure [14].

\section{Anti-Atherosclerotic Effects Beyond Glucose Lowering of Dapagliflozin}

Improvement in glucose control, body weight and blood pressure by dapagliflozin was almost confirmed by a relatively large number of studies. We hypothesized the underlying mechanisms for possible anti-atherosclerotic effects beyond glucose lowering of SGLT-2 inhibitors (Fig. 1). We searched the published articles about the effects of dapagliflozin on metabolic risk factors by using Pubmed (Table 2). In this search, we excluded "Original Articles using animals or cells", "Narrative Reviews" and "Expert Opinions", and we considered "Original Articles", Systematic Reviews" and "Meta-analysis" as important information.

\section{The effect of dapagliflozin on visceral or body fat}

Patients $(\mathrm{N}=182)$ were randomly assigned to receive dapagliflozin $10 \mathrm{mg}$ /day or placebo added to open-label metformin

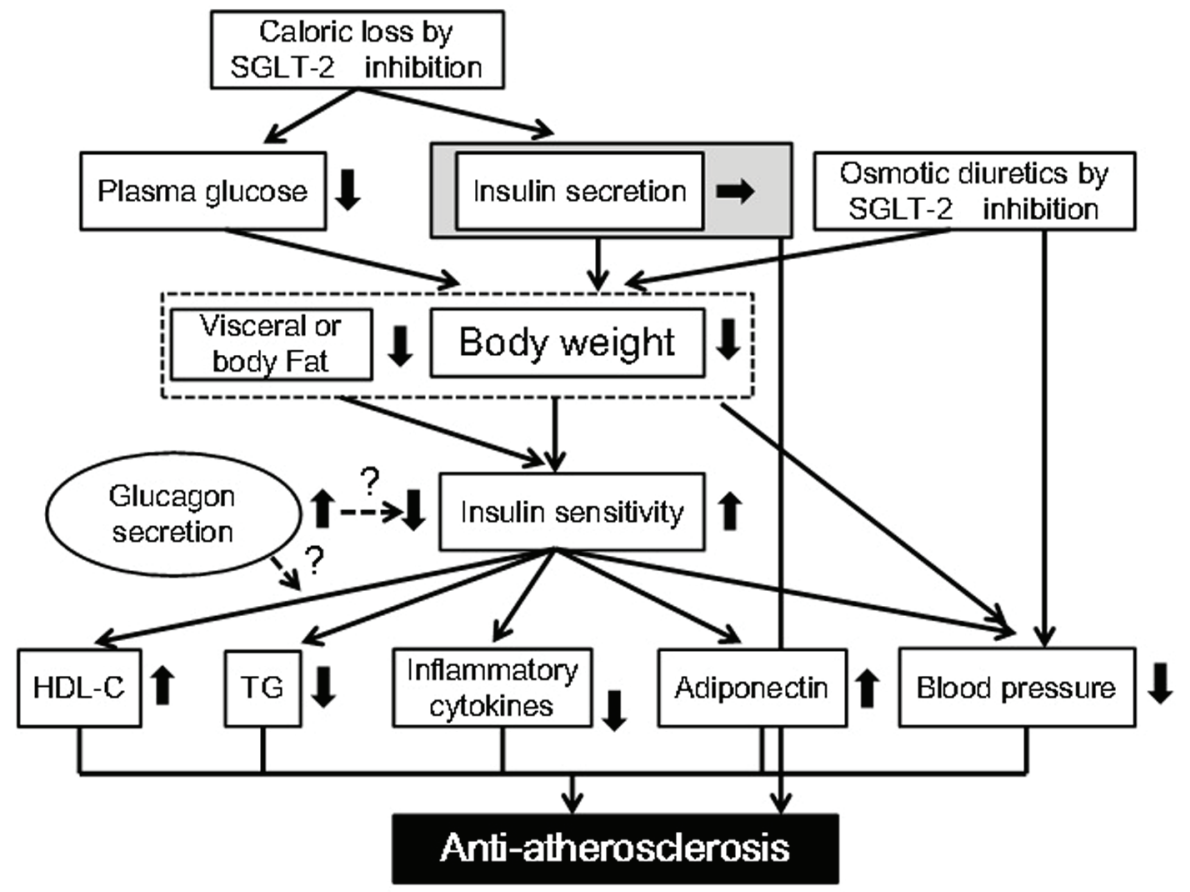

Figure 1. Possible anti-atherosclerotic effects beyond glucose lowering of sodium glucose cotransporter 2 inhibitors. HDL-C: high-density lipoprotein-cholesterol; SGLT-2: sodium glucose cotransporter 2; TG: triglyceride. 
Table 2. The Search Strategy to Find the Anti-Atherosclerotic Effects of Dapagliflozin

\begin{tabular}{ll}
\hline The search strategy by using Pubmed & Published articles (n) \\
\hline Dapagliflozin and body weight & 106 \\
Dapagliflozin and blood pressure & 78 \\
$\begin{array}{l}\text { Dapagliflozin and body fat OR dapagliflozin and visceral fat OR dapagliflozin } \\
\text { and waist circumference OR dapagliflozin and abdominal circumference }\end{array}$ & 4 \\
Dapagliflozin and insulin resistance OR dapagliflozin and insulin sensitivity & 38 \\
Dapagliflozin and low density lipoprotein OR dapagliflozin and LDL & 5 \\
Dapagliflozin and high density lipoprotein OR dapagliflozin and HDL & 6 \\
Dapagliflozin and triglyceride & 4 \\
Dapagliflozin and adiponectin & 0 \\
Dapagliflozin and CRP OR dapagliflozin and C-reactive protein & 1 \\
Dapagliflozin and tumor necrosis factor alpha OR dapagliflozin and TNF- $\alpha$ & 0 \\
Dapagliflozin and interleukin-6 OR dapagliflozin and IL-6 & 0 \\
Dapagliflozin and cytokine OR dapagliflozin and cytokines & 2 \\
\hline
\end{tabular}

[15]. Over 102 weeks, dapagliflozin-treated patients showed reductions in waist circumference by $-5.0 \mathrm{~cm}$ and fat mass by $-2.8 \mathrm{~kg}$. In another study, 182 patients with diabetes were allocated to receive dapagliflozin $10 \mathrm{mg} /$ day or placebo added to open-label metformin for 24 weeks [16]. Placebo-corrected changes with dapagliflozin were as follows: waist circumference, $-1.52 \mathrm{~cm}(95 \% \mathrm{CI}=-2.74$ to $-0.31 ; \mathrm{P}=0.0143)$; fat mass, $-1.48 \mathrm{~kg}(95 \% \mathrm{CI}=-2.22$ to $-0.74 ; \mathrm{P}=0.0001)$; visceral adipose tissue, $-258.4 \mathrm{~cm}^{3}(95 \% \mathrm{CI}=-448.1$ to -68.6 ; nominal $\mathrm{P}$ $=0.0084)$.

\section{The effect of dapagliflozin on insulin sensitivity}

We selected "Original Articles" which evaluated insulin sensitivity using the euglycemic hyperinsulinemic clamp. Twentyfour subjects with diabetes received dapagliflozin $(\mathrm{n}=16)$ or placebo $(\mathrm{n}=8)$ for 2 weeks, and the euglycemic hyperinsulinemic clamp was performed before and after treatment [17]. Dapagliflozin significantly improved whole-body insulin sensitivity. In another study, 18 diabetic men were randomized to receive either dapagliflozin $(n=12)$ or placebo $(n=6)$ for 2 weeks [18]. Improvement in muscle insulin sensitivity by dapagliflozin was observed by using the euglycemic hyperinsulinemic clamp. Forty-four subjects were randomized to receive dapagliflozin $5 \mathrm{mg}$ or matching placebo once daily for 12 weeks [19]. Insulin sensitivity was assessed by measuring the glucose disappearance rate during the last 40 min of a 5-h euglycemic hyperinsulinemic clamp. Dapagliflozin treatment improved insulin sensitivity.

\section{The effect of dapagliflozin on serum lipids}

In the study by Matthaei et al [12], adjusted \% changes of fasting LDL-cholesterol from baseline to week $52(95 \% \mathrm{CI})$ in the placebo group and the dapagliflozin group were $0.9(-6.7,9.1)$ and $4.8(-1.5,11.5)$, respectively. Adjusted \% changes in fast- ing HDL-cholesterol were $0.6(-3.6,4.9)$ and $6.9(3.3,10.6)$ in the placebo group and the dapagliflozin group, respectively. Adjusted \% changes in fasting LDL/HDL ratio were 0.9 (-7.8, $10.5)$ and $-2.5(-9.3,4.7)$. Adjusted \% changes in fasting triglyceride were $2.9(-8.1,15.2)$ and $-8.0(-16.0,0.7)$. This study suggested that dapagliflozin is beneficially associated with serum lipids. Matthaei et al also evaluated the efficacy and safety of a 24-week dapagliflozin treatment in patients with type 2 diabetes inadequately controlled with metformin and sulfonylurea [20]. Patients receiving dapagliflozin showed placebosubtracted increases in total, LDL, and HDL-cholesterol (11.4 $\mathrm{mg} / \mathrm{dL}, \mathrm{P}=0.0091 ; 11.4 \mathrm{mg} / \mathrm{dL}, \mathrm{P}=0.0030 ; 2.2 \mathrm{mg} / \mathrm{dL}, \mathrm{P}=$ 0.0172 , respectively) with no change in LDL/HDL ratio (0.1; $\mathrm{P}=0.2008)$ or triglycerides $(-16.5 \mathrm{mg} / \mathrm{dL} ; \mathrm{P}=0.1755)$. This study did not show evident beneficial effects of dapagliflozin for serum lipids, except for an increase of HDL-cholesterol. In a meta-analysis of randomized clinical trials, SGLT-2 inhibitors determined a modest but statistically significant increase in HDL-cholesterol, with no effect on triglyceride and on total and LDL-cholesterol [14].

\section{The effect of dapagliflozin on inflammation and adipocy- tokines}

In the review which critically assessed the results of up-todate clinical trials with dapagliflozin [21], it was described that high sensitivity C-reactive protein levels were decreased in dapagliflozin-treated patients. We could not find published articles about effects of dapagliflozin on adipocytokines including interleukin-6, tumor necrosis factor alpha and adiponectin.

\section{The effect of dapagliflozin on glucagon secretion}

In the study by Merovci et al [18], insulin-mediated tissue glucose disposal increased by approximately $18 \%$ after 2 -week dapagliflozin treatment, while placebo-treated subjects had no 
change in insulin sensitivity. Following dapagliflozin treatment, an increase in fasting plasma glucagon concentration was observed. Glucagon is associated with insulin resistance [22]. An influence of increase of glucagon secretion by SGLT2 inhibitors on insulin resistance or metabolic risk factors should be carefully observed.

\section{Conclusion}

SGLT-2 inhibitors seem to be associated with weight loss and reduction of blood pressure by a relatively large number of studies. However, the studies that investigated effects of dapagliflozin on visceral fat, insulin sensitivity, serum lipids, inflammation and adipocytokines are very limited. Furthermore, an influence of increase in glucagon secretion by SGLT2 inhibitors on metabolic risk factors remains unknown. The glucose lowering effect in an insulin-independent manner of SGLT-2 inhibitors prevents hyperinsulinemia, which may also contribute to anti-atherogenesis. Very recently, Zinman et al showed that the addition of another SGLT-2 inhibitor, empagliflozin, reduced rates of death from cardiovascular causes $(38 \%$ relative risk reduction), hospitalization for heart failure (35\%), and death from any cause (32\%) [23], suggesting a possible anti-atherosclerotic effect of SGLT-2 inhibitors. However, further studies should be performed to elucidate anti-atherosclerotic effect of SGLT-2 inhibitors.

\section{Competing Interests}

The authors declare that they have no competing interests.

\section{Funding}

This work was funded by a grant from the National Center for Global Health and Medicine (26-112).

\section{References}

1. Vallon V, Platt KA, Cunard R, Schroth J, Whaley J, Thomson SC, Koepsell H, et al. SGLT2 mediates glucose reabsorption in the early proximal tubule. J Am Soc Nephrol. 2011;22(1):104-112.

2. Jabbour SA, Goldstein BJ. Sodium glucose co-transporter 2 inhibitors: blocking renal tubular reabsorption of glucose to improve glycaemic control in patients with diabetes. Int J Clin Pract. 2008;62(8):1279-1284.

3. Savage PJ. Cardiovascular complications of diabetes mellitus: what we know and what we need to know about their prevention. Ann Intern Med. 1996;124(1 Pt 2):123-126.

4. Stamler J, Vaccaro O, Neaton JD, Wentworth D. Diabetes, other risk factors, and 12-yr cardiovascular mortality for men screened in the Multiple Risk Factor Intervention Trial. Diabetes Care. 1993;16(2):434-444.

5. Kannel WB, McGee DL. Diabetes and cardiovascular dis- ease. The Framingham study. JAMA. 1979;241(19):20352038.

6. Fuller JH, Shipley MJ, Rose G, Jarrett RJ, Keen H. Mortality from coronary heart disease and stroke in relation to degree of glycaemia: the Whitehall study. Br Med J (Clin Res Ed). 1983;287(6396):867-870.

7. Barrett-Connor EL, Cohn BA, Wingard DL, Edelstein SL. Why is diabetes mellitus a stronger risk factor for fatal ischemic heart disease in women than in men? The Rancho Bernardo Study. JAMA. 1991;265(5):627-631.

8. Goldbourt U, Yaari S, Medalie JH. Factors predictive of long-term coronary heart disease mortality among 10,059 male Israeli civil servants and municipal employees. A 23-year mortality follow-up in the Israeli Ischemic Heart Disease Study. Cardiology. 1993;82(2-3):100-121.

9. Manson JE, Colditz GA, Stampfer MJ, Willett WC, Krolewski AS, Rosner B, Arky RA, et al. A prospective study of maturity-onset diabetes mellitus and risk of coronary heart disease and stroke in women. Arch Intern Med. 1991;151(6):1141-1147.

10. Haffner SM, Lehto S, Ronnemaa T, Pyorala K, Laakso M. Mortality from coronary heart disease in subjects with type 2 diabetes and in nondiabetic subjects with and without prior myocardial infarction. N Engl J Med. 1998;339(4):229-234.

11. Oliva RV, Bakris GL. Blood pressure effects of sodiumglucose co-transport 2 (SGLT2) inhibitors. J Am Soc Hypertens. 2014;8(5):330-339.

12. Matthaei S, Bowering K, Rohwedder K, Sugg J, Parikh $\mathrm{S}$, Johnsson E. Durability and tolerability of dapagliflozin over 52 weeks as add-on to metformin and sulphonylurea in type 2 diabetes. Diabetes Obes Metab. 2015;17(11):1075-1084.

13. Kaku K, Maegawa H, Tanizawa Y, Kiyosue A, Ide Y, Tokudome T, Hoshino Y, et al. Dapagliflozin as monotherapy or combination therapy in Japanese patients with type 2 diabetes: an open-label study. Diabetes Ther. 2014;5(2):415-433.

14. Monami M, Nardini C, Mannucci E. Efficacy and safety of sodium glucose co-transport-2 inhibitors in type 2 diabetes: a meta-analysis of randomized clinical trials. Diabetes Obes Metab. 2014;16(5):457-466.

15. Bolinder J, Ljunggren O, Johansson L, Wilding J, Langkilde AM, Sjostrom CD, Sugg J, et al. Dapagliflozin maintains glycaemic control while reducing weight and body fat mass over 2 years in patients with type 2 diabetes mellitus inadequately controlled on metformin. Diabetes Obes Metab. 2014;16(2):159-169.

16. Bolinder J, Ljunggren O, Kullberg J, Johansson L, Wilding J, Langkilde AM, Sugg J, et al. Effects of dapagliflozin on body weight, total fat mass, and regional adipose tissue distribution in patients with type 2 diabetes mellitus with inadequate glycemic control on metformin. J Clin Endocrinol Metab. 2012;97(3):1020-1031.

17. Merovci A, Mari A, Solis C, Xiong J, Daniele G, ChavezVelazquez A, Tripathy D, et al. Dapagliflozin lowers plasma glucose concentration and improves beta-cell function. J Clin Endocrinol Metab. 2015;100(5):1927-1932.

18. Merovci A, Solis-Herrera C, Daniele G, Eldor R, Fiorenti- 
no TV, Tripathy D, Xiong J, et al. Dapagliflozin improves muscle insulin sensitivity but enhances endogenous glucose production. J Clin Invest. 2014;124(2):509-514.

19. Mudaliar S, Henry RR, Boden G, Smith S, Chalamandaris AG, Duchesne D, Iqbal N, et al. Changes in insulin sensitivity and insulin secretion with the sodium glucose cotransporter 2 inhibitor dapagliflozin. Diabetes Technol Ther. 2014;16(3):137-144.

20. Matthaei S, Bowering K, Rohwedder K, Grohl A, Parikh $\mathrm{S}$. Dapagliflozin improves glycemic control and reduces body weight as add-on therapy to metformin plus sul- fonylurea: a 24-week randomized, double-blind clinical trial. Diabetes Care. 2015;38(3):365-372.

21. Katsiki N, Papanas N, Mikhailidis DP. Dapagliflozin: more than just another oral glucose-lowering agent? Expert Opin Investig Drugs. 2010;19(12):1581-1589.

22. Eaton RP. Evolving role of glucagon in human diabetes mellitus. Diabetes. 1975;24(5):523-524.

23. Zinman B, Wanner C, Lachin JM, Fitchett D, Bluhmki E, Hantel S, Mattheus M, et al. Empagliflozin, Cardiovascular Outcomes, and Mortality in Type 2 Diabetes. N Engl J Med. 2015. 